\title{
Dopamine Transporter Correlates and Occupancy by Modafinil in Cocaine-Dependent Patients: A Controlled Study With High-Resolution PET and [ ' ${ }^{\text {C }]-P E 2 I ~}$
}

\author{
Laurent Karila*,1,2, Claire Leroy ${ }^{1,3,4}$, Manon Dubol', Christian Trichard ${ }^{1,5}$, Audrey Mabondo', \\ Catherine Marill' ${ }^{2}$, Albertine Dubois ${ }^{6}$, Nadège Bordas', Jean-Luc Martinot ${ }^{1,7}$, Michel Reynaud ${ }^{2,7}$ and \\ Eric Artiges $1,5,7$
}

'INSERM U. 1000 Research Unit 'Neuroimaging and Psychiatry', Paris Sud University, Paris Descartes University, Paris, Orsay, France; ${ }^{2}$ AP-HP, Addiction Research and Treatment Center, Paul Brousse Hospital, Villejuif, France; ${ }^{3}$ CEA, DSV, I2BM, Service Hospitalier Frédéric Joliot, Orsay, France; ${ }^{4}$ Laboratoire Imagerie Moléculaire In Vivo (IMIV), CEA, Inserm, Paris Sud University, CNRS, Université Paris Saclay, CEA-SHFJ, Orsay, France; ${ }^{5}$ Psychiatry Department, Orsay Hospital, Orsay, France; ' Laboratoire Imagerie et Modélisation en Neurobiologie et Cancérologie, UMR 8 I 65 CNRS-Université Paris 7-Université Paris II, Orsay, France

Modafinil is a candidate compound for the treatment of cocaine addiction that binds to the dopamine transporter (DAT) in healthy humans, as observed by positron emission tomography (PET). This mechanism, analogous to that of cocaine, might mediate a putative therapeutic effect of modafinil on cocaine dependence, though the binding of modafinil to DAT has never been assessed in cocainedependent patients. We aimed at quantifying the DAT availability during a controlled treatment by modafinil, and its clinical and psychometric correlates in cocaine-dependent patients at the onset of abstinence initiation. Twenty-nine cocaine-dependent male patients were enrolled in a 3-month trial for cocaine abstinence. Modafinil was used in a randomized double-blind placebo-controlled design and was administered as follows: 400 mg/day for 26 days, then 300 mg/day for 30 days, and 200 mg/day for 31 days. Participants were examined twice during a 17-day hospitalization for their DAT availability using PET and [ ' C]-PE2I and for assessments of craving, depressive symptoms, working memory, and decision-making. Cocaine abstinence was further assessed during a I0-week outpatient follow-up period. Baseline [ $\left.{ }^{\prime \prime} \mathrm{C}\right]-\mathrm{PE} 2 \mathrm{l}$-binding potential covaried with risk taking and craving index in striatal and extrastriatal regions. A $65.6 \%$ decrease of binding potential was detected in patients receiving modafinil for 2 weeks, whereas placebo induced no significant change. During hospitalization, an equivalent improvement in clinical outcomes was observed in both treatment groups, and during the outpatient follow-up there were more therapeutic failures in the modafinil-treated group. Therefore, these results do not support the usefulness of modafinil to treat cocaine addiction.

Neuropsychopharmacology (2016) 4I, 2294-2302; doi:I0.1038/npp.20 I6.28; published online I6 March 2016

\section{INTRODUCTION}

Cocaine dependence is a significant worldwide public health issue with somatic, psychiatric, cognitive, socio-economic, and legal complications (Karila et al, 2011a). As almost all psychoactive drugs causing addiction in humans, cocaine activates the dopaminergic meso-cortico-limbic pathways (Feltenstein and See, 2008). Cocaine acts as a reuptake inhibitor for the monoamine neurotransmitters and its particular action on the dopamine transporter (DAT), known to regulate dopamine transmission, is usually considered as critical for its psychoactive effects and the establishment of an addiction (Volkow et al, 2006).

* Correspondence: Dr L Karila, AP-HP, Addiction Research and Treatment Centre, Paul Brousse Hospital, 12 Avenue Paul Vaillant Couturier, Villejuif, 94800, France, Tel: +33 I 455965 13, E-mail: laurent.karila@aphp.fr

${ }^{7}$ These authors contributed equally to this work.

Received 4 October 2015; revised 3 February 2016; accepted 7 February 2016; accepted article preview online 9 February 2016
Furthermore, chronic use of cocaine is known to have a major effect on DAT density in dependent patients. Indeed, the majority of postmortem and imaging studies reported increases of DAT levels in cocaine users suggesting an adaptive change of DAT density, although some others reported decreases or no change (Narendran and Martinez, 2008). In the absence of cocaine, DAT augmentation could produce a relative dopamine deficiency and contribute to cocaine-seeking behavior and relapse (Volkow et al, 2004).

Currently, no specific pharmacotherapy has established its efficacy in treating cocaine dependence and the development of new medications continues to be a research priority. Among several promising pharmacological approaches, modafinil emerges as a reasonable candidate (Karila et al, 2011a). Modafinil is a non-amphetamine stimulant, approved for the treatment of sleep disorders such as narcolepsy. In recent years, modafinil has shown potential in the treatment of cocaine dependence (Mariani and Levin, 2012) 
both in laboratory self-administration and double-blind, placebo-controlled clinical studies (Dackis et al, 2005; Hart et al, 2008), although positive results may be limited to subpopulations of cocaine-dependent patients (Anderson et al, 2009; Dackis et al, 2012). The neurobiological mechanism of modafinil is complex as it produces appreciable effects on noradrenergic, GABAergic, and glutamatergic neurotransmissions (Ferraro et al, 1998; Madras et al, 2006; Volkow et al, 2009). Modafinil has also a low micromolar affinity for the DAT (Mereu et al, 2013) as compared with the nanomolar affinity of cocaine. Recently, positron emission tomography (PET) studies with modafinil reported a significant DAT blockade and increased extracellular dopamine levels in humans and non-human primates (Madras et al, 2006; Volkow et al, 2009; Andersen et al, 2010; Kim, 2012). At effective doses in narcolepsy, the level of DAT blockade in humans is around $50 \%$ in the dorsal striatum and slightly less in the nucleus accumbens (Volkow et al, 2009; Kim, 2012). Taken together, these data suggest that the potential therapeutic effect of modafinil in cocaine dependence could be mediated by its action on the DAT. However, this remains to be determined in cocaine users, as well as the link between the DAT blockade and the therapeutic effects of modafinil.

In this study, we used high-resolution PET imaging and a specific DAT radioligand, $\left[{ }^{11} \mathrm{C}\right]-\mathrm{N}$-(3-iodopro-2E-enyl)2beta-carbomethoxy-3beta-(4'-methylphenyl) nortropane $\left(\left[{ }^{11} \mathrm{C}\right]-\mathrm{PE} 2 \mathrm{I}\right)$, to investigate cerebral DAT availability in cocaine-dependent inpatients. Participants were examined twice, before and after 2 weeks of treatment, within the framework of a 3-month longitudinal randomized modafinil ( $400 \mathrm{mg}$ /day) vs placebo-controlled study. The main aims of the study were, first, to evaluate the level of DAT occupancy induced by therapeutic dosage of modafinil in cocaine users, and second, to examine whether DAT availability was correlated with the clinical assessments of the patients (craving, depressive symptoms, or cognitive functions). Another aim of the study was to provide further data on modafinil efficacy in the treatment of cocaine dependence.

\section{MATERIALS AND METHODS}

\section{Study Design}

The present study is a 3-month randomized double-blind study using brain imaging and modafinil $400 \mathrm{mg}$ /day vs placebo (Figure 1). Enrolled cocaine-dependent patients were hospitalized from day 0 to day 17 . They were examined twice, before and after 2 weeks of treatment (on days 3 and 17). The participants went through magnetic resonance imaging (MRI)/PET imaging, neuropsychological tests, and clinical and biological assessments (craving and depression rating, blood tests, and urinary toxicology screening tests). From day 4 to day 90 , modafinil $100 \mathrm{mg}$ tablets or placebo

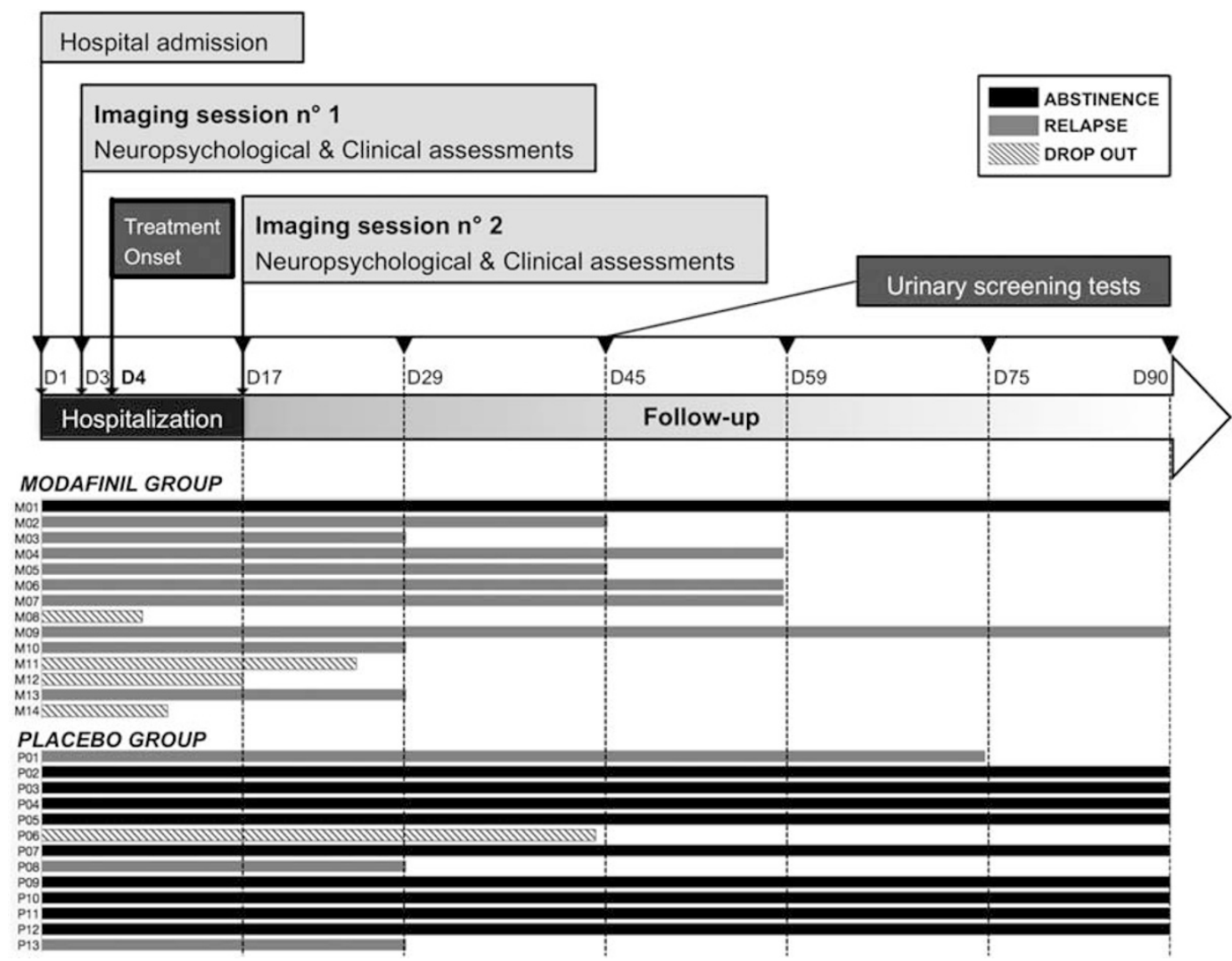

Figure I Study design and patients' clinical course. During hospitalization, neuroimaging session, neuropsychological tests (N-back; lowa Gambling task), clinical assessments (craving and depression ratings), and urinary toxicology screening tests were performed twice. After hospitalization, patients came back every 2 weeks for medical consultation, and urine samples were collected to define abstinence or relapse. The decrease of modafinil dosage occurred on day 30 (400-300 mg) and day 60 (300-200 mg). The horizontal bars represent the clinical course of each participant throughout the study. 
tablets were orally administered in the morning as follows: four tablets for 26 days, then three tablets for 30 days, and finally two tablets for 31 days. A cocaine abstinence followup was carried out from day 17 to day 90 using urinary benzoylecgonine (BE) dosage. Urine samples were collected on days $29,45,59,75$, and 90 to assess abstinence after hospitalization. Thereafter the treatment was discontinued. Compliance with medication was measured by pill count using the treatment blisters. Each group was blind to modafinil or placebo until the end of the study. The study was registered as the Cocaine Addiction Imaging Medications and Neurotransmitters (CAIMAN) study, with ClinicalTrials.gov identifier NCT00701532.

\section{Participants}

The study was approved by the local ethics committee (Comité de Protection des Personnes Ile-de-France 7). Oral and written consent were obtained after complete description of the study. Participants were randomized by the Department of Clinical Research of Paris Sud University.

Twenty-nine cocaine-dependent male patients aged 26-52 years were recruited in the Cocaine Reference Center of Paul Brousse University Hospital, Villejuif (France) over a period of 2 months. All patients met DSM-IV criteria for cocaine dependence, had a positive urine toxicology screen for cocaine in the weeks prior to hospitalization, were seeking treatment, and were willing to give informed consent prior to participation. Exclusion criteria were: a known hypersensitivity to modafinil or modafinil contraindications, any substance-related Axis I disorder (except tobacco dependence), any treatment that interferes with the DAT or modafinil, and MRI contraindications.

\section{Clinical Assessments}

Depression was assessed using the Montgomery-Asberg Depression Rating Scale (MADRS) (Montgomery and Asberg, 1979) and the Beck Depression Inventory (BDI) (Beck et al, 1961). Craving was evaluated using a seven-point visual analog scale, the brief version of the Cocaine Craving Questionnaire (10-item CCQ brief) measuring the current craving (Karila et al, 2011b). Cognitive deficits were measured using two neuropsychological tests: Bechara's Iowa Gambling Task (IGT) (Bechara et al, 1994) assessing decision-making, and a visuospatial N-Back task assessing working memory (Carlson et al, 1998). These tests were carried out in session by a senior neuropsychologist.

\section{Pharmacological Treatments}

Modafinil (100 mg tablets) is chemically and pharmacologically distinct from other central nervous system stimulants and is approved for the treatment of sleep disorders. The pharmacokinetics of modafinil is linear, independent of the administered dose. Peak plasma concentrations are reached $2-3 \mathrm{~h}$ after its use. The elimination half-life of modafinil is 10-12 $\mathrm{h}$. The pharmacodynamic activity does not seem to affect the autonomic nervous system and the cardiovascular system. The usual dosage for this treatment is $200-400 \mathrm{mg}$ per day as a single dose in the morning. It has numerous side effects and some contraindications (FDA, 2007). The placebo tablets were identical to the modafinil ones. In case of withdrawal symptoms, hydroxyzine $25 \mathrm{mg}$ were administered three times per day.

\section{Image Acquisition and Processing}

PET images were acquired on a Siemens ECAT HRRT 3D-PET scanner (CPS innovations Services, Knoxville, TN, USA). Previous studies using $\left[{ }^{11} \mathrm{C}\right]-\mathrm{PE} 2 \mathrm{I}$ and HRRT in healthy subjects reported good suitability and test/retest reproducibility, and better accuracy in quantifying DAT binding, as compared with conventional PET scanners (Hirvonen et al, 2008). $\left[{ }^{11} \mathrm{C}\right]-\mathrm{PE} 2 \mathrm{I}$ was prepared using a TRACERlab FX-C Pro synthesizer (Gems, Velisy, France). The acquisition started with the bolus injection of $300 \mathrm{MBq}$ of $\left[{ }^{11} \mathrm{C}\right]$-PE2I and lasted $60 \mathrm{~min}$ (20 sequential frames from 1 to $5 \mathrm{~min}$ were acquired). Images were reconstructed using the statistical algorithm ordinary Poisson-ordered subset expectation maximization. The voxel size was $1.2 \times 1.2 \times$ $1.2 \mathrm{~mm}^{3}$. The injected radioactivity for $\left[{ }^{11} \mathrm{C}\right]-\mathrm{PE} 2 \mathrm{I}$ was $306.3 \pm 56.0 \mathrm{MBq}$ in scan 1 (day 3 ) and $319.46 \pm 38.35 \mathrm{MBq}$ in scan 2 (day 17). The specific radioactivity in the two scans was $29.3 \pm 12.8$ and $28.6 \pm 14.0 \mathrm{MBq} / \mu \mathrm{mol}$, respectively. There was no difference between the groups on days 3 and 17 in injected radioactivity $(\mathrm{F}(1,26)=0.01, P=0.91$ and $\mathrm{F}(1,21)=1.33, P=0.26$, respectively) or in specific radioactivity $(\mathrm{F}(1,26)=0.69, P=0.41$ and $\mathrm{F}(1,21)=1.16, P=0.29$, respectively).

MRI was acquired on a 1.5-T Signa scanner (General Electric Healthcare, Milwauke, WI, USA). A T1-weighted sequence was performed using the following MRI parameters: 3D Fourier-transform spoiled-gradient-recalled acquisition with $\mathrm{TR}=12.5 \mathrm{~ms}$, $\mathrm{TE}=2.2 \mathrm{~ms}, 124$ contiguous slices of $1.3 \mathrm{~mm}$ thickness, a field of view of $24 \mathrm{~cm}$, and a $256 \times 256$ view matrix, with a voxel size of $0.9 \times 0.9 \times$ $1.3 \mathrm{~mm}^{3}, 16 \mathrm{bits} /$ pixel.

Motion corrections during PET acquisition were carried out using a home-made tool within the BrainVISA/ Anatomist software (http://brainvisa.info) consisting in frame by frame co-registration of the PET dynamic series using a mutual information method. Thereafter, in order to process parametric binding potential images, brain regions were determined by T1-MRI automatic parcellation and applied on dynamic co-registered PET images using the PMOD PNEURO tool, version 3.4 (PMOD, Zurich, Switzerland). Time-activity curves obtained from bilateral dorsal caudate and putamen nuclei as high specific binding and crus1 sub-region of the cerebellum as a reference tissue were exported to PMOD's pixel-wise tool. Parametric maps of the regional $\left[{ }^{11} \mathrm{C}\right]$-PE2I non-displaceable binding potential $\left(\mathrm{BP}_{\mathrm{ND}}\right)$ were generated using Gunn's basis function method (Gunn et al, 1997), which is closely related to the simplified reference tissue model (Lammertsma and Hume, 1996). Previous studies have confirmed the suitability of specific $\left[{ }^{11} \mathrm{C}\right]-$ PE2I binding quantification using a compartmental approach with the cerebellum as reference region (Seki et al, 2010). Spatial normalization was applied on the $\mathrm{BP}_{\mathrm{ND}}$ maps using SPM8 (Statistical Parametric Mapping, Welcome Department of Cognitive Neurology, London, UK) with a ligand-specific $\left[{ }^{11} \mathrm{C}\right]$-PE2I template generated according to an MRI-aided procedure (Meyer et al, 1999). The normalized 
$\mathrm{BP}_{\mathrm{ND}}$ maps were smoothed using a $10-\mathrm{mm}$ FWHM Gaussian filter. The voxel size was $2 \times 2 \times 2 \mathrm{~mm}^{3}$.

\section{Statistical Analysis}

Among the 29 patients enrolled, two left the study on the first day of hospitalization and were excluded from the analysis. Twenty-seven patients were compared on enrolment on demographic and clinical characteristics using Student's $t$-test. In order to assess their course during hospitalization, we performed repeated-measures analyses of variance on clinical and neuropsychological data between day 3 and day 17 . These analyses included time (day 3, day 17) as withinsubject factor and group (modafinil, placebo) as betweensubject factor.

During follow-up, samples containing BE at concentrations $\geqslant 300 \mathrm{ng} / \mathrm{ml}$ were considered positive for cocaine. Therapeutic failures were defined by dropouts or at least one cocaine-positive urine sample, and abstinence was defined by negative urine samples. A chi-square test (Fisher's Exact test) was used to compare the treatment groups in terms of therapeutic failure and abstinence throughout the study for 27 participants. We used post-hoc t-tests on day 3 clinical data to ensure that the therapeutic failures and the abstinent patients were not clinically different at the beginning of the study. JMP 10 (JMP, SAS Institute, Cary, NC, 1989-2007) was used to perform these analyses.

PET images voxel-wise analyses were performed with SPM8. A one-sample $t$-test of $\mathrm{BP}_{\mathrm{ND}}$ maps acquired on day 3 was performed to visualize the localization of DAT availability in the brain before treatment and was used to define a mask of analysis. Analyses were conducted within a brain mask defined as all voxels with a minimum value of PET signal, which was $50 \%$ superior to the maximum value of the crus1 area (cerebellar reference region). This mask included bilateral striatum, insula, pallidum, claustrum, amygdala, thalamus, midbrain, inferior frontal cortex (gyrus rectus, olfactory cortex and orbitofrontal cortex) and temporal cortex (hippocampus, parahippocampal gyrus).

We compared $\mathrm{BP}_{\mathrm{ND}}$ maps using a repeated-measures analysis of variance (flexible factorial design in SPM8) with subjects and time as within-subject factors and group as between-subject factor. This analysis was conducted to examine the global group effect, the time effect and the group by time interaction for the patients who were present to the two imaging sessions $(N=22)$. Post-hoc two-sample $t$-tests were used to specify the differences between treatment groups on day 3 and day 17 separately, with age as confounding covariate. Likewise, post-hoc paired $t$-tests were used to assess time effect between day 3 and day 17 within the two groups separately.

Correlation analyses were performed between $\mathrm{BP}_{\mathrm{ND}}$ images and clinical or neuropsychological variables (craving and depression rates, working memory and decision-making scores, and urinary $\mathrm{BE}$ amounts) using multiple regression analyses, with age as confounding covariate. Analyses were conducted across all patients on day $3(n=27)$ and the two groups on day 17 ( $n=9$ and $n=13$ for the modafinil and placebo groups, respectively).

In order to compare the radiotracer binding in the treatment groups before and after treatment, averaged PET signal was extracted from the significant cluster of the interaction analysis using the MarsBaR toolbox implemented in SPM8 (Brett et al, 2002). We calculated the percentage of $\mathrm{BP}_{\mathrm{ND}}$ difference $\left(\left(\left(\mathrm{BP}_{\mathrm{ND}(\text { day3 })}-\mathrm{BP}_{\mathrm{ND}(\text { day } 17)}\right) / \mathrm{BP}_{\mathrm{ND}(\text { day } 3)}\right) \times\right.$ $100)$ between day 3 and day 17 using the averaged signals extracted by the MarsBar toolbox. We compared the averaged $\mathrm{BP}_{\mathrm{ND}}$ values between group and time conditions using $t$-tests.

\section{RESULTS}

\section{Demographic and Clinical Results}

Table 1 summarizes demographic characteristics and clinical measures of the two groups on the day of enrolment (day 0 ). There were no significant differences for age, alcohol and tobacco use, cocaine addiction characteristics, or urinary BE amount between the treatment groups.

During hospitalization, three patients dropped out prior to the day-17 imaging session and two did not go through PET scan on day 17 owing to technical failure issues (dysfunctions in HRRT scanner and in $\left[{ }^{11} \mathrm{C}\right]-\mathrm{PE} 2 \mathrm{I}$ synthesis). These five patients were in the modafinil group. Therefore, the analyses

Table I Participants' Characteristics on Day 0

\begin{tabular}{|c|c|c|c|c|}
\hline Characteristics & placebo $(n=13)$ & modafinil $(n=14)$ & t-Value & $t$-Test (P-values) \\
\hline Age (years) & $37.8(6.8)$ & $37.5(5.7)$ & 0.11 & 0.91 \\
\hline Alcohol use (g/week) & $131.5(124.5)$ & $223.6(226)$ & 1.3 & 0.21 \\
\hline Tobacco use (cigarettes/day) & $17.2(7.7)$ & $14.5(10.8)$ & 0.74 & 0.46 \\
\hline Age at the first contact with cocaine (years) & $25.1(6.2)$ & $24.5(3.2)$ & 0.34 & 0.73 \\
\hline Age when cocaine use became regular (years) & $31.5(8.2)$ & $31.2(5.5)$ & 0.12 & 0.9 \\
\hline Amount of cocaine use (g/week) & $12.9(9.7)$ & $8.0(9.7)$ & 1.4 & 0.17 \\
\hline Weekly cocaine cost $(€)$ & $749.2(608.8)$ & $568.9(638.3)$ & 0.74 & 0.46 \\
\hline Days between consecutive cocaine uses & $3.2(2.5)$ & $2.5(1.9)$ & 0.83 & 0.41 \\
\hline Last cocaine use before hospitalization (days) & $5.5(4.4)$ & $2.2(3.8)$ & 2.07 & 0.05 \\
\hline Urinary cocaine amount on Day $0(\mathrm{BE}, \mathrm{ng} / \mathrm{ml})^{\mathrm{a}}$ & $4894.1(3418.3)$ & $9073.1(12167.2)$ & 1.19 & 0.24 \\
\hline
\end{tabular}

Means (SD). Two-sample t-tests revealed no significant difference between the groups on day 0 .

arine samples containing benzoylecgonine (BE) at concentrations $>300 \mathrm{ng} / \mathrm{ml}$ are considered to be positive for cocaine. 

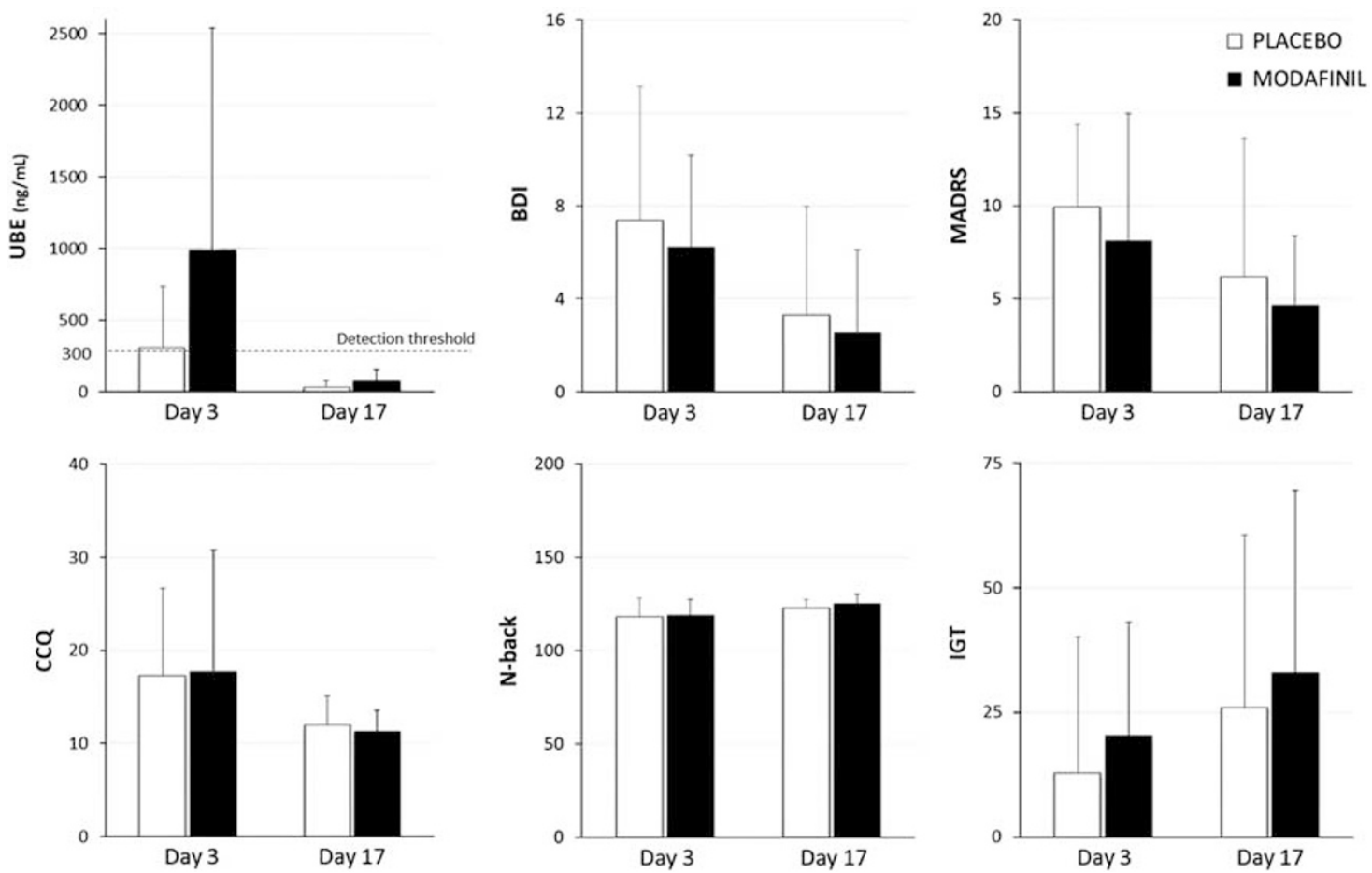

Figure 2 Clinical and neuropsychological outcomes during hospitalization. Repeated-measures analyses showed only significant time effects. No significant time $\times$ group interaction or group effect were found. BDI, Beck depression inventory; CCQ, cocaine craving questionnaire; ITG, lowa gambling task score; MADRS, Montgomery and Asberg depression rating scale; N-Back, N-back memory task score; UBE, urinary benzoylecgonine (BE) dosage (urine samples containing BE at concentrations $>300 \mathrm{ng} / \mathrm{ml}$ are considered to be positive for cocaine). Data are reported as means+SD.

on day 17 included 9 participants in the modafinil group and 13 participants in the placebo group.

Repeated-measures analyses of variance revealed a significant time effect in both groups, showing a decrease in urinary $\mathrm{BE}$ amount between day 3 and day $17(P=0.016)$, craving scores $(P=0.026)$, and depression rates (MADRS, $P=0.0003$ and BDI, $P<0.0001)$ and an improvement of memory skills $(P=0.003)$. The analysis also indicates a trend toward improved IGT scores $(P=0.049)$ over the days of hospitalization (Figure 2, Supplementary Table S1). Regarding the patients' clinical course during hospitalization, no difference was found between the modafinil and the placebo groups.

After hospitalization, the modafinil group displayed more therapeutic failures than the placebo group, ie, more dropouts and cocaine-positive urine samples, $(76.5 \%, n=13$ vs $23.5 \%, n=4)$ and less abstinent patients $(10 \%, n=1 v s$ $90 \%, n=9)\left(\chi^{2}\right.$ Fisher's Exact test, $\left.\mathrm{df}=1, P=0.0013\right)$. Indeed, over the 3-month period of the study, 13 of the 17 failures received modafinil while 9 of the 10 abstinent patients received placebo. The clinical course of each participant is described in Figure 1. The post-hoc comparison between therapeutic failures and abstinent patients revealed that their clinical data did not differ at the beginning of the study (data not shown).

Among all the participants, 8 were treated by serotoninnorepinephrine reuptake inhibitors or selective serotonin reuptake inhibitors treatments, and 22 took hydroxyzine pills during the study. Hydroxyzine or antidepressant treatments were not related to therapeutic failure or abstinence (Fisher's Exact test, $P=1$ and $P=0.36$, respectively).

\section{Imaging Results}

Analysis of $\mathrm{BP}_{\mathrm{ND}}$ revealed a significant interaction between time and group factors in a bilateral symmetric pattern (Table 2). The two-sample $t$-tests revealed no group effect on the $\mathrm{BP}_{\mathrm{ND}}$ values on day 3 but a significant group effect on day 17 , showing lower $\mathrm{BP}_{\mathrm{ND}}$ values in the modafinil group in comparison with the placebo group, after 2 weeks of treatment. These differences were located bilaterally in the striatum (caudate and putamen), insula, hippocampus, amygdala, globus pallidus, and midbrain (substantia nigra and ventral tegmental area) (Supplementary Table S2). The paired $t$-tests showed a significant time effect in the modafinil group, denoting a decrease of $\mathrm{BP}_{\mathrm{ND}}$ values between day 3 and day 17 in amygdala, hippocampus, insula, striatum, and midbrain (Supplementary Table S3). There was no significant time effect in the placebo group.

The averaged PET signal quantification revealed a significant time effect for the modafinil-treated patients only (Figure 3). Indeed, the modafinil group showed a $65.6 \%$ mean decrease of $\mathrm{BP}_{\mathrm{ND}}$ between day 3 and day 17. Detailed regional $\mathrm{BP}_{\mathrm{ND}}$ values in the striatal and midbrain areas are shown in Supplementary Table S4.

A multiple regression analysis on the 27 patients on day 3 showed correlations between $\mathrm{BP}_{\mathrm{ND}}$ and behavioral data. IGT scores correlated negatively $\left(P<0.05_{\mathrm{FWE}}\right.$ corrected $)$ with $\mathrm{BP}_{\mathrm{ND}}$ in the striatum, thalamus, insula, and globus pallidus (Supplementary Table S5). Craving scores correlated positively $\left(P<0.005_{\text {uncorrected }}\right)$ with $\mathrm{BP}_{\mathrm{ND}}$ in the striatum, gyrus rectus and olfactory cortex, globus pallidus, and insula, exclusively in the left hemisphere (Supplementary Table S6). 
Table 2 Regional Analysis of Time (Day 3, Day 17) by Group (Modafinil, Placebo) Interaction Using Statistical Parametric Mapping (SPM 8)

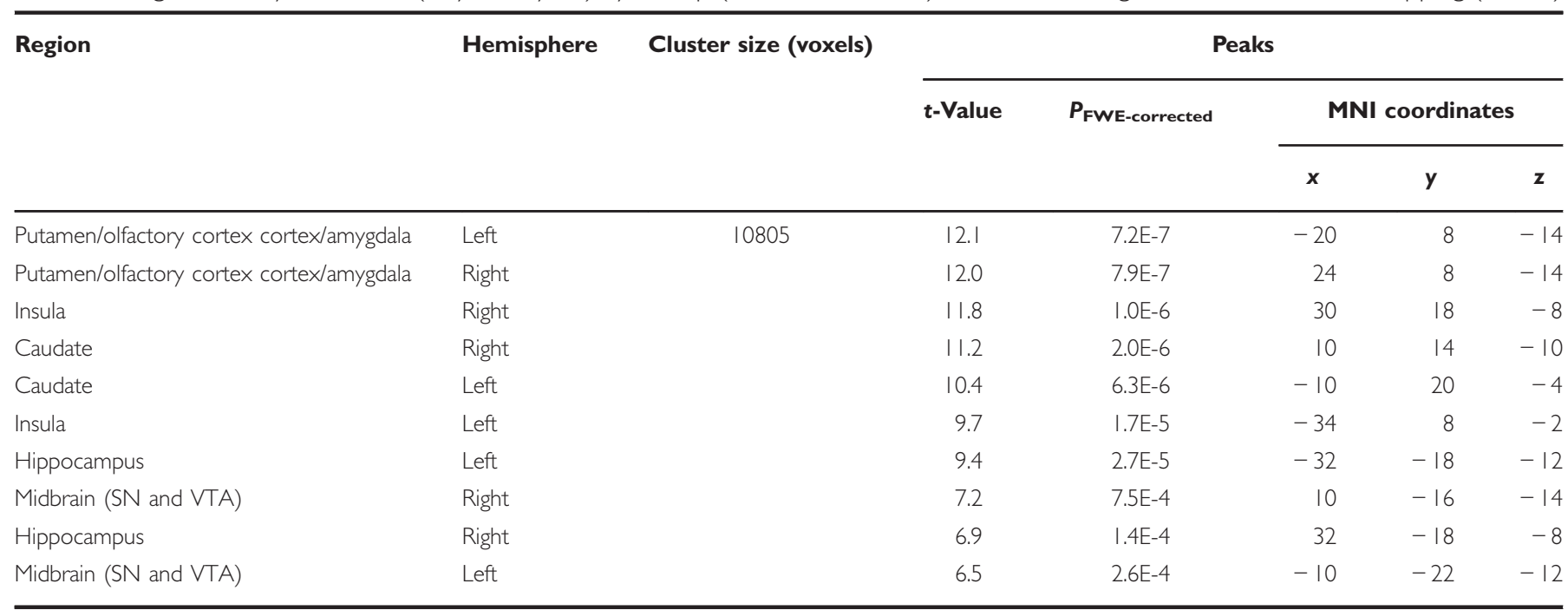

Abbreviations: SN, substantia nigra; VTA, ventral tegmental area. Height and extent thresholds set at $P<0.05$ family wise-error (FWE) corrected. Anatomical regions were determined from the Montreal Neurological Institute (MNI) coordinates.
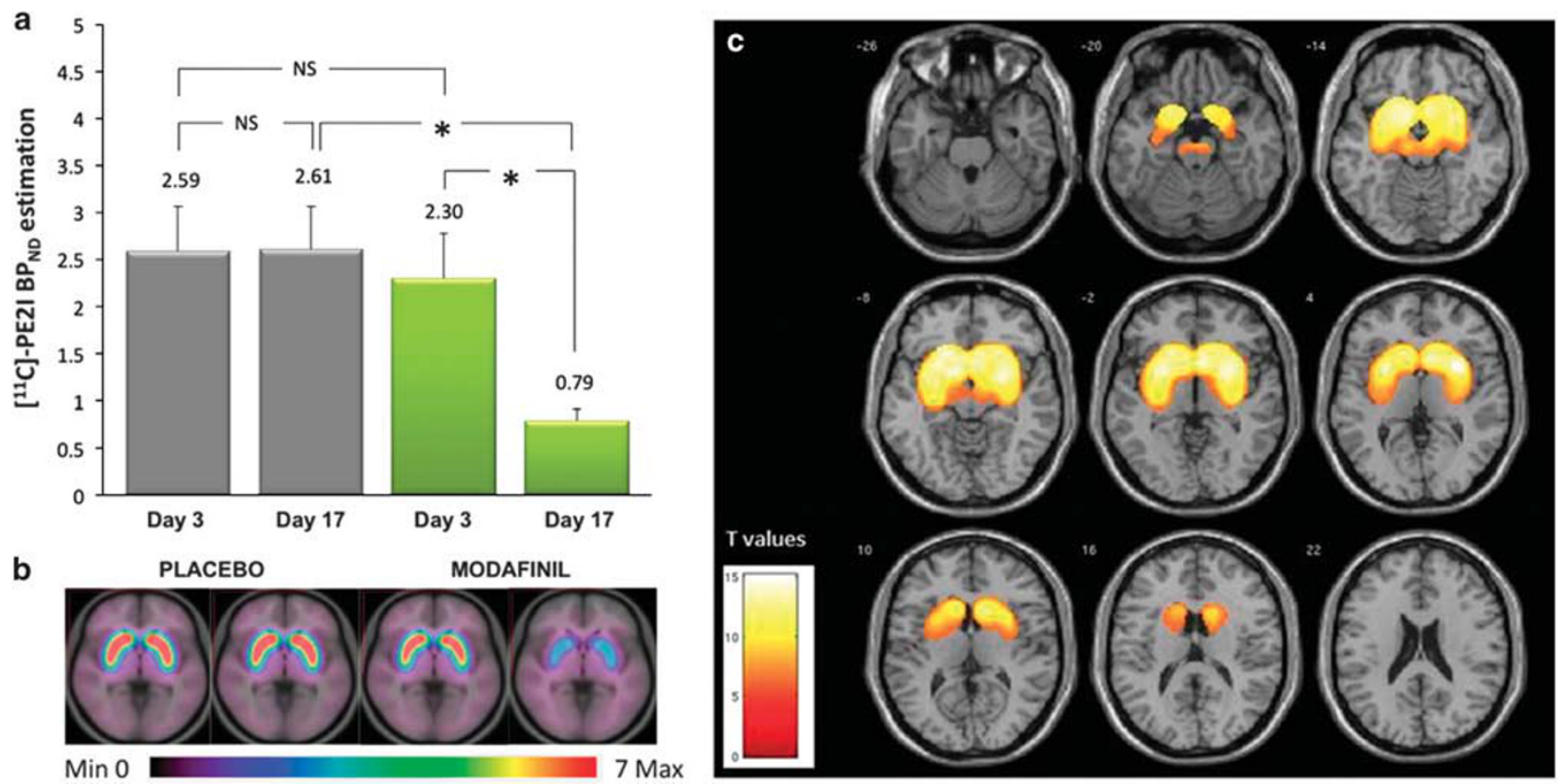

Figure 3 Imaging results. (a). Graph represents the global averaged PET signal extracted from the significant cluster of the interaction analysis. Error bars indicate \pm SD. *Significant difference. NS, not significant. (b) Averaged [I I C]-PE2I BP ${ }_{\text {ND }}$ maps, overlaid onto a MRI Template, from the placebo and modafinil groups on days 3 and 17. (c) Statistical parametric map of $\mathrm{BP}_{\mathrm{ND}}$ obtained from the modafinil<placebo contrast on day 17.

We did not find any correlation between $\mathrm{BP}_{\mathrm{ND}}$ and depression rates, memory scores, or urinary $\mathrm{BE}$ amounts on day 3 nor any correlation between $\mathrm{BP}_{\mathrm{ND}}$ and clinical data on day 17 (either for the placebo group or for the modafinil group).

\section{DISCUSSION}

The current investigation established the high level of DAT blockade induced by modafinil in cocaine-dependent patients enrolled in a double-blind controlled trial. Before treatment, we also detected correlations between DAT availability and both cocaine craving and performance to a risk-taking task. Despite its effect on the DAT, modafinil failed to show an actual advantage vs placebo during hospitalization. Furthermore, modafinil was associated with more therapeutic failures at follow-up.

\section{Neuroimaging and Correlates}

The regional distribution of the radiotracer binding was consistent with the localization of the DAT previously 
reported from human brain autoradiography and PET studies using $\left[{ }^{11} \mathrm{C}\right]-\mathrm{PE} 2 \mathrm{I}$ and $\left[{ }^{11} \mathrm{C}\right]$-cocaine (Marcusson and Eriksson, 1988; Sekine et al, 2003; Tupala et al, 2006; Taber et al, 2012). Here the 400-mg daily dosage of modafinil inhibited the $\left[{ }^{11} \mathrm{C}\right]$-PE2I radiotracer binding on the DAT by about $65 \%$ in cocaine-dependent patients. This result is in line with studies in healthy subjects that reported $51-57 \%$ mean striatal DAT occupancy with 200-400 mg single doses of modafinil (Volkow et al, 2009; Kim et al, 2014). Differences in design, as the single or repeated administration or the $200-400 \mathrm{mg}$ dosage of modafinil, might explain the slightly more elevated DAT blockade in the present study. In the placebo group, no significant difference of DAT availability was detected between day 3 and day 17 , supporting that the abstinence period between the two PET scans was too short to observe any regulation of the DAT availability. This adds further evidence that the decreased $\left[{ }^{11} \mathrm{C}\right]-\mathrm{PE} 2 \mathrm{I}$ binding in the modafinil group basically reflects the DAT occupancy.

Decision-making impairments have been highlighted previously in drug abusers using neuropsychological tasks, such as the IGT (Grant et al, 2000). We detected a negative correlation between the IGT scores and the DAT availability on day 3 in regions of the reward system such as the striatum and the thalamus and in the insula. Hence, the higher the DAT availability in these regions, the lower the IGT score. Considering that IGT low scores reflect immediate rewardoriented strategies and risky choices, this adds to evidence of a relationship between decision-making dysfunction and dopamine activity in cocaine-dependent patients (Bechara and Damasio, 2002; Balconi et al, 2014). This is also in line with a recent study showing that risky decision-making can be modulated by dopaminergic drugs in healthy subjects (Norbury et al, 2013).

Such a relationship was also reported with the insula, a region involved in decision-making and cognitive control in cocaine-dependent patients (Naqvi and Bechara, 2009; Cisler et al, 2013). As the insula is mostly known to integrate interoceptive information into conscious feelings and decision-making processes, it has been hypothesized that interoceptive effects of drugs influence the reward system through processes involving dopamine within the insula (Ernst and Paulus, 2005; Naqvi and Bechara, 2009). Consistent with this hypothesis, our results support a relationship between dopamine, insula, and decisionmaking in cocaine-dependent patients.

Furthermore, the scores of craving correlated positively with $\left[{ }^{11} \mathrm{C}\right]-\mathrm{PE} 2 \mathrm{I} \mathrm{BP}_{\mathrm{ND}}$ on day 3 in the left striatum, ventromedial prefrontal cortex, and insula. Interestingly, although uncorrected this left prevailing laterality is consistent with previous studies showing an involvement of dopamine in cocaine craving, in the left dorsal striatum (Volkow et al, 2006; Wong et al, 2006). Neural activity within the insula has been shown to be related to craving (Koob and Le Moal, 2001; Li et al, 2010), and ventromedial parts of the prefrontal cortex are known to represent the subjective value of rewards and goals and drive goal-directed behavior (O'Doherty, 2011). Thus our observations in cocainedependent patients abstinent for 3 days further highlight the role of the DAT in these regions in addiction.

\section{Clinical Outcomes}

At the end of hospitalization, the modafinil and placebo groups displayed an equivalent improvement of clinical and neuropsychological outcomes. No therapeutic advantage of modafinil $400 \mathrm{mg}$ /day was detected during hospitalization on cocaine craving, withdrawal-induced depressive symptoms, and cognitive performances. The follow-up period even suggested a worsening effect of modafinil in achieving abstinence. Indeed, modafinil treatment was associated with more BE-positive urine samples and dropouts than the placebo. Additionally, the three patients who voluntary dropped out during hospitalization (prior to day 17) all belonged to the modafinil group. Interestingly, clinical characteristics of the therapeutic failures and abstinent patients did not significantly differ in the beginning of the study (data not shown), further supporting the involvement of modafinil in this outcome.

This finding is quite surprising in the light of encouraging previous studies showing that modafinil promotes cocaine abstinence (Dackis et al, 2005; Kampman et al, 2015) and reduces cocaine craving and withdrawal (Karila et al, 2011a). Nevertheless, other studies have displayed more contrasting results, suggesting that beneficial effects of modafinil on cocaine abstinence could be limited to sub-populations of patients (Anderson et al, 2009; Dackis et al, 2012). To our knowledge, our study is the first to suggest a deleterious effect of modafinil in cocaine-dependent patients. The reasons of this observation are unclear. However, several characteristics of our study could be involved in this finding. First, the current study design was basically intended for PET imaging. Consequently, it enrolled a small sample, limiting the statistical power of our clinical results. Second, previously published controlled studies included only outpatients while in our study cocaine-dependent patients were hospitalized during the first 17 days. Thus it is likely that our patients were particularly motivated to achieve abstinence as they accepted both the imaging and hospitalization constraints. This certainly had a substantial effect on the high abstinence rate in the placebo group. Last, it cannot be excluded that the modafinil dosage of $400 \mathrm{mg} /$ day we used was not optimal to achieve abstinence in some patients (Anderson et al, 2009).

\section{Limitations}

There are some limitations in this present work. First, although the small sample size was comparable to other brain imaging studies, clinical outcomes should be cautiously interpreted. It also precluded us from testing for different dosages of modafinil. Second, based on previous studies, we decided to include only men without alcohol comorbidity (Anderson et al, 2009; Dackis et al, 2012). Thus our results cannot be extended to all cocaine users. Third, there are some technical limitations regarding PET imaging. Although 60-min PET acquisition appears sufficient for extrastriatal DAT imaging, this short acquisition period might affect the quantification of DAT binding in high-density regions, such as the striatum (Hirvonen et al, 2008; Seki et al, 2010). However, owing to the longitudinal design of the study, this bias should not have significantly modified the direction of the findings. Moreover, we detected DAT availability in cortical-limbic regions such as hippocampus, insula, and 
orbital-frontal, where DAT protein was previously reported (Marcusson and Eriksson, 1988; Sekine et al, 2003; Tupala et al, 2006). As the fraction of specific binding in those regions (specific binding/total binding) is low (10-15\%), small variations in non-specific binding may impact the $\mathrm{BP}_{\mathrm{ND}}$. Therefore, significant correlations with $\mathrm{BP}_{\mathrm{ND}}$ values in corticolimbic areas should be considered with caution.

In conclusion, relationships between DAT availability and indices of craving or propensity to risk taking were detected for the first time in cocaine-dependent patients. We also confirm in these patients the high level of DAT occupancy induced by a daily dose of $400 \mathrm{mg} /$ day of modafinil, similarly as previously reported in healthy subjects (Volkow et al, 2009; Kim et al, 2014). In our study, modafinil did not promote abstinence in cocaine addiction.

\section{FUNDING AND DISCLOSURE}

The Cocaine Addiction Imaging Medications And Neurotransmitters (CAIMAN) study was supported by grants from the French Ministry of Health (PHRC 2007) and from MILDECA-INCa-INSERM (2006). The authors declare no conflict of interest.

\section{ACKNOWLEDGMENTS}

The research was monitored by the Unité de Recherche Clinique des Hôpitaux Universitaires Paris Sud (Professor Laurent Becquemont). We thank Ms Christine Baron, Mr Claude Comtat, Mr Frédéric Dollé, Ms Rose-Marie Dubuisson, Mr Philippe Gervais, and Mr Renaud Maroy from the Service Hospitalier Frédéric Joliot for their efficient technical support.

\section{REFERENCES}

Andersen ML, Kessler E, Murnane KS, McClung JC, Tufik S, Howell LL (2010). Dopamine transporter-related effects of modafinil in rhesus monkeys. Psychopharmacology 210: 439-448.

Anderson AL, Reid MS, Li SH, Holmes T, Shemanski L, Slee A et al (2009). Modafinil for the treatment of cocaine dependence. Drug Alcohol Depend 104(1-2): 133-139.

Balconi M, Finocchiaro R, Canavesio Y (2014). Reward-system effect (BAS rating), left hemispheric "unbalance" (alpha band oscillations) and decisional impairments in drug addiction. Addict Behav 39: 1026-1032.

Bechara A, Damasio AR, Damasio H, Anderson SW (1994). Insensitivity to future consequences following damage to human prefrontal cortex. Cognition 50(1-3): 7-15.

Bechara A, Damasio H (2002). Decision-making and addiction (part I): impaired activation of somatic states in substance dependent individuals when pondering decisions with negative future consequences. Neuropsychologia 40: 1675-1689.

Beck AT, Ward CH, Mendelson M, Mock J, Erbaugh J (1961). An inventory for measuring depression. Arch Gen Psychiatry 4: 561-571.

Brett M, Anton J, Valabregue R, Poline J-B (2002). Region of interest analysis using an SPM toolbox. The 8th International Conference on Functional Mapping of the Human Brain; Sendai, Japan. June 2-6, 2002.

Carlson S, Martinkauppi S, Rama P, Salli E, Korvenoja A, Aronen HJ (1998). Distribution of cortical activation during visuospatial n-back tasks as revealed by functional magnetic resonance imaging. Cereb Cortex 8: 743-752.

Cisler JM, Elton A, Kennedy AP, Young J, Smitherman S, Andrew James $G$ et al (2013). Altered functional connectivity of the insular cortex across prefrontal networks in cocaine addiction. Psychiatry Res 213: 39-46.

Dackis CA, Kampman KM, Lynch KG, Pettinati HM, O'Brien CP (2005). A double-blind, placebo-controlled trial of modafinil for cocaine dependence. Neuropsychopharmacology 30: 205-211.

Dackis CA, Kampman KM, Lynch KG, Plebani JG, Pettinati HM, Sparkman T et al (2012). A double-blind, placebo-controlled trial of modafinil for cocaine dependence. J Subst Abuse Treat 43: 303-312.

Ernst M, Paulus MP (2005). Neurobiology of decision making: a selective review from a neurocognitive and clinical perspective. Biol Psychiatry 58: 597-604.

FDA (2007). Food and Drug Administration. Patient information leaflet for PROVIGIL" (modafinil) Tablet [C-IV].

Feltenstein MW, See RE (2008). The neurocircuitry of addiction: an overview. Br J Pharmacology 154: 261-274.

Ferraro L, Antonelli T, O'Connor WT, Tanganelli S, Rambert FA, Fuxe K (1998). The effects of modafinil on striatal, pallidal and nigral GABA and glutamate release in the conscious rat: evidence for a preferential inhibition of striato-pallidal GABA transmission. Neurosci Lett 253: 135-138.

Grant S, Contoreggi C, London ED (2000). Drug abusers show impaired performance in a laboratory test of decision making. Neuropsychologia 38: 1180-1187.

Gunn RN, Lammertsma AA, Hume SP, Cunningham VJ (1997). Parametric imaging of ligand-receptor binding in PET using a simplified reference region model. Neuroimage 6: 279-287.

Hart CL, Haney M, Vosburg SK, Rubin E, Foltin RW (2008). Smoked cocaine self-administration is decreased by modafinil. Neuropsychopharmacology 33: 761-768.

Hirvonen J, Johansson J, Teras M, Oikonen V, Lumme V, Virsu P et al (2008). Measurement of striatal and extrastriatal dopamine transporter binding with high-resolution PET and [11C]PE2I: quantitative modeling and test-retest reproducibility. J Cereb Blood Flow Metab 28: 1059-1069.

Kampman KM, Lynch KG, Pettinati HM, Spratt K, Wierzbicki MR, Dackis C et al (2015). A double blind, placebo controlled trial of modafinil for the treatment of cocaine dependence without co-morbid alcohol dependence. Drug Alcohol Depend 155: 105-110.

Karila L, Reynaud M, Aubin HJ, Rolland B, Guardia D, Cottencin O et al (2011a). Pharmacological treatments for cocaine dependence: is there something new? Curr Pharm Des 17: 1359-1368.

Karila L, Seringe E, Benyamina A, Reynaud M (2011b). The reliability and validity of the French version of the Cocaine Craving Questionnaire-Brief. Curr Pharm Des 17: 1369-1375.

Kim D (2012). Practical use and risk of modafinil, a novel waking drug. Environ Health Toxicol 27: e2012007.

Kim W, Tateno A, Arakawa R, Sakayori T, Ikeda Y, Suzuki H et al (2014). In vivo activity of modafinil on dopamine transporter measured with positron emission tomography and [(1)(8)F]FE-PE2I. Int J Neuropsychopharmacol 17: 697-703.

Koob GF, Le Moal M (2001). Drug addiction, dysregulation of reward, and allostasis. Neuropsychopharmacology 24: 97-129.

Lammertsma AA, Hume SP (1996). Simplified reference tissue model for PET receptor studies. Neuroimage 4(3 Pt 1): 153-158.

Li CS, Luo X, Sinha R, Rounsaville BJ, Carroll KM, Malison RT et al (2010). Increased error-related thalamic activity during early compared to late cocaine abstinence. Drug Alcohol Depend 109(1-3): 181-189.

Madras BK, Xie Z, Lin Z, Jassen A, Panas H, Lynch L et al (2006). Modafinil occupies dopamine and norepinephrine transporters in vivo and modulates the transporters and trace amine activity in vitro. J Pharmacol Exp Ther 319: 561-569. 
Marcusson J, Eriksson K (1988). [3H]GBR-12935 binding to dopamine uptake sites in the human brain. Brain Res 457: 122-129.

Mariani JJ, Levin FR (2012). Psychostimulant treatment of cocaine dependence. Psychiatr Clin North Am 35: 425-439.

Mereu M, Bonci A, Newman AH, Tanda G (2013). The neurobiology of modafinil as an enhancer of cognitive performance and a potential treatment for substance use disorders. Psychopharmacology 229: 415-434.

Meyer JH, Gunn RN, Myers R, Grasby PM (1999). Assessment of spatial normalization of PET ligand images using ligand-specific templates. Neuroimage 9: 545-553.

Montgomery SA, Asberg M (1979). A new depression scale designed to be sensitive to change. Br J Psychiatry 134: 382-389.

Naqvi NH, Bechara A (2009). The hidden island of addiction: the insula. Trends Neurosci 32: 56-67.

Narendran R, Martinez D (2008). Cocaine abuse and sensitization of striatal dopamine transmission: a critical review of the preclinical and clinical imaging literature. Synapse 62: 851-869.

Norbury A, Manohar S, Rogers RD, Husain M (2013). Dopamine modulates risk-taking as a function of baseline sensationseeking trait. J Neurosci 33: 12982-12986.

O'Doherty JP (2011). Contributions of the ventromedial prefrontal cortex to goal-directed action selection. Ann NY Acad Sci 1239: $118-129$.

Seki C, Ito H, Ichimiya T, Arakawa R, Ikoma Y, Shidahara M et al (2010). Quantitative analysis of dopamine transporters in human brain using [11C]PE2I and positron emission tomography: evaluation of reference tissue models. Ann Nuclear Med 24: 249-260.

Sekine Y, Minabe Y, Ouchi Y, Takei N, Iyo M, Nakamura K et al (2003). Association of dopamine transporter loss in the orbitofrontal and dorsolateral prefrontal cortices with methamphetaminerelated psychiatric symptoms. Am J Psychiatry 160: 1699-1701.

Taber KH, Black DN, Porrino LJ, Hurley RA (2012). Neuroanatomy of dopamine: reward and addiction. J Neuropsychiatry Clin Neurosci 24: 1-4.

Tupala E, Halonen P, Tiihonen J (2006). Visualization of the cortical dopamine transporter in type 1 and 2 alcoholics with human whole hemisphere autoradiography. Eur Neuropsychopharmacol 16: 552-560.

Volkow ND, Fowler JS, Logan J, Alexoff D, Zhu W, Telang F et al (2009). Effects of modafinil on dopamine and dopamine transporters in the male human brain: clinical implications. JAMA 301: 1148-1154.

Volkow ND, Fowler JS, Wang GJ (2004). The addicted human brain viewed in the light of imaging studies: brain circuits and treatment strategies. Neuropharmacology 47(Suppl 1): 3-13.

Volkow ND, Wang GJ, Telang F, Fowler JS, Logan J, Childress AR et al (2006). Cocaine cues and dopamine in dorsal striatum: mechanism of craving in cocaine addiction. I Neurosci 26: 6583-6588.

Wong DF, Kuwabara H, Schretlen DJ, Bonson KR, Zhou Y, Nandi A et al (2006). Increased occupancy of dopamine receptors in human striatum during cue-elicited cocaine craving. Neuropsychopharmacology 31: 2716-2727.

Supplementary Information accompanies the paper on the Neuropsychopharmacology website (http://www.nature.com/npp) 